\title{
Facing Provider Misconceptions Towards the Use of Hormone Therapy in $\mathbf{2 0 2 0}$
}

\author{
Heather Hirsch, MD, MS, NCMP D
}

Department of Internal Medicine, Division of Women's Health, Menopause \& Midlife Clinic, Brigham and Women's Hospital, Harvard Medical School, 75 Francis Street, Boston, MA, USA.

$\mathrm{J}$ Gen Intern Med 36(3):767-8

DOI: $10.1007 / \mathrm{s} 11606-020-05940-\mathrm{w}$

(c) Society of General Internal Medicine 2020

\section{PERSPECTIVE PIECE}

The New England Journal of Medicine recently published a clinical vignette highlighting the treatment of menopause by Dr. JoAnn V. Pinkerton, former North American Menopause Society (NAMS) executive director and president. ${ }^{1}$ The vignette highlighted that hormone therapy (HT) should be considered first-line therapy for vasomotor symptom (VMS) treatment in women who are less than ten years from menopause and who have no known contraindications to HT use. However, what still needs to be addressed is the need for dialogue surrounding the misconceptions that physicians and laywomen have about hormone therapy. Since 2002 when results of the Women's Health Initiative (WHI) first hit the front pages of newspapers, the evening news, and physicians' inboxes, a deep-rooted idea has persisted throughout US culture that estrogen is dangerous and harmful despite significant evidence to the contrary. ${ }^{2-4}$

As a fellowship-trained NAMS-certified internist who practices consultative menopause, I consistently see the look of astonishment in my resident's eyes when they see me prescribe HT. I watch my patients hesitantly walk out of my office with their prescription in hand. My trainees quickly learn the gratitude that my patients have for my specialized education, medical skills, and persistency in deconstructing the social myths when they return with an improvement in their symptoms. So, despite nearly a decade of reports on the benefits of HT outweighing the risks, ${ }^{5}$ why have not we moved the needle much when it comes to easing providers and patients fears of estrogen?

Unnecessary harm has been inflicted upon millions of women when they are denied HT or worse, when physicians themselves perpetuate inaccurate facts. To open this dialogue, I have identified three areas where physicians and laywomen may have been misguided, and a call to action for each of these.

First, the barriers begin with the fact that members of society (physicians and laypersons alike) are bombarded with information on menopause that is often inconsistent, inaccurate, and/or

Received March 12, 2020

Accepted May 20, 2020

Published online June 4, 2020 harmful. This may misguide physicians on the appropriate use of FDA-approved treatment options available and considered first line. ${ }^{6}$ A leading source of disinformation is the unregulated, non-FDA-approved HT industry. These compounded hormone products are not recommended by medical societies, including NAMS, the American College of Obstetrics and Gynecology (ACOG), and the Endocrine Society. Yet, this highly profitable market is thriving by claiming to be "safer" than FDA-approved options. Previously collected data concerning unregulated, compounded HT use has shown numerous risks to women including uterine hyperplasia, uterine cancer, and supratherapeutic testosterone levels. ${ }^{7}$ Moreover, these treatments incur significant financial out of pocket expenses and unnecessary lab testing. Ultimately, physicians do harm by not explicating correcting this major source of disinformation. ${ }^{8}$

Unlike in the UK or other countries with greater regulation of medications and supplements, authorized providers in the USA have the autonomy to prescribe unregulated HT, or to avoid discussing or using HT as first-line therapy for women facing menopausal-related symptoms. In contrast, in the UK, the National Institute for Health and Care Excellence (NICE) guidelines set protocols physicians must follow. Recently the NICE guidelines have been updated to include HT as first-line treatment for women with symptomatic VMS or genitourinary syndrome of menopause (GSM). ${ }^{9}$ While this approach is not translatable to US medical practice, some oversight may now be necessary considering the harms that are occurring to women in the USA who are being offered unconventional HT options, or not being offered FDA-approved treatment. ${ }^{10}$ The call to action is this: physicians must attempt to present a clear, consistent, and evidence-based message about the safety of FDA-approved HT options, and discourage unregulated HT options. This could mean that medical societies and possibly state licensure departments attempt to hold providers responsible when not following consensus guidelines. To avoid further confusion, the data for estrogen alone and estrogen plus progestogen therapy should be split and the effects in women should be reported separately, due to their differing benefits, side effects, and risk profiles. ${ }^{11}$

Secondly, medical and gynecological residents have inadequate training (or postgraduate re-training) in caring for women experiencing menopausal symptoms. ${ }^{12,}{ }^{13}$ It remains unclear why residency training is not equipped to adequately prepare new physicians to feel comfortable prescribing HT 
upon graduation, but may be related to the decline in HT use in the early $2000 \mathrm{~s},{ }^{14}$ and the resulting lack of accompanying exposure received from residents in training. In 2019, a cross-sectional study was undertaken to evaluate knowledge regarding HT education received by undergraduates in family medicine, internal medicine, and OB/GYN. Out of 177 participants, $6.8 \%$ reported feeling properly prepared to manage menopausal complaints. ${ }^{15}$ This demonstrates that although the risk-benefit ratio for women who start HT less than ten years from menopause is particularly favorable, ${ }^{1}$ a lack of training may be a significant factor in the proper treatment of restoring quality of life in menopausal women. ${ }^{12}$ The call to action is this: Physicians, especially those in training, need experience and exposure through focused medical school and residency training programs, and all primary care trainees should receive substantial menopause education. As it currently stands, the American College of Graduate Medical Education (ACGME) does not have clear standards for menopause training, leaving room for change, especially as trainees in nearly every specialty of medicine will encounter a menopausal-related complaint in their career. Furthermore, continued (re)education on menopause training can be supported at large-scale meetings such as the American College of Physicians (ACP) or the Society of General Internal Medicine (SGIM), not just at specialty meetings such as NAMS.

Lastly, in Western culture, menopause is given little exposure, affecting the way menopause is experienced by women at home, in the workplace, by the media, and at the doctor's office. ${ }^{16}$ For many women, midlife is intensely challenging, which means these parties have a responsibility to represent the menopause transition as seriously as they do other common health conditions that receive more traditional exposure. Interestingly, grassroots organizations of women across the globe are starting to fight for the establishment of workplace policies to protect women going through menopause. And, major networks like NBC have recently tackled the topic of menopause and sexual health at midlife. For my part, I often recommend my patients bring along their partner to their visits, which may change how menopause is experienced at home. For their part, physicians can refamiliarize themselves with the pathophysiology of menopause and its distinct intersection with chronic disease management, ${ }^{17}$ and alterations in quality of life.

So, while challenging cultural norms is seemingly out of a physician's singular control, the call to action is this: Physicians can start by recognizing and challenging personal, medical, and social misconceptions surrounding menopause management and treatment. Physicians, who have an undeniable impact on changing community health needs, can help spark the momentum for others to ignite change and demand more from their social support networks, employers, and the media.

In conclusion, nearly every practicing physician in the USA is going to encounter menopause in their practice, and this fact makes it imperative that we begin to reshape and challenge our misconceptions around estrogen replacement and/or face our lack of training head on. In the meantime, we can look inwards by examining how we would treat our mothers, sisters, and daughters if they were our menopausal patient.

Corresponding Author: Heather Hirsch, MD, MS, NCMP; Department of Internal Medicine, Division of Women's Health, Menopause \& Midlife Clinic, Brigham and Women's Hospital, Harvard Medical School, 75 Francis Street, Boston, MA 02115, USA (e-mail: hhirsch@bwh.harvard.edu).

\section{Compliance with Ethical Standards:}

Conflict of Interest: The author has no conflicts of interest.

\section{REFERENCES}

1. Pinkerton V JoAnn. Hormone Therapy for Postmenopausal Women. NEJM. 2020.

2. Hoffmann M, Hammar M, Kiellgren KL et al. Change in women's attitudes towards and use of hormone therapy after HERS and WHI. Maturitas. 2005;52(1):11-7.

3. Huston SA, Kirking DM, Shimp LA. Use, intentions, and beliefs about hormone replacement therapy in women with and without diabetes. Marturitas. 2006;55(1):58-68.

4. Sivan T, Carbon J, Ross S, Yuksel N. Determinants of hormone therapy uptake and decision-making after bilateral oophorectomy (BO): A narrative review. Maturitas. 2019;120:68-76.

5. Gurney EP, Nachtigall MJ, Nachtigall LE. The Women's Health Initiative trial and related studies: 10 years later: a clinician's view. J Steriod Biochem Mol Biol. 2014;142:4-11.

6. Taylor S Hugh, Kagan R, Altomare, C. et al. Knowledge of clinical trials regarding hormone therapy and likelihood of prescribing hormone therapy. Menopause. Vol 24. No 1. pp27-34.

7. Gass ML, Stuenkel CA, Utian WH. Use of compounded hormone therapy in the United States: report of The North American Menopause Society Survey. Menopause. 22(12): 1276-1285, 2015.

8. Thompson JJ, Ritenbaugh $\mathbf{C}$, Nichter $\mathbf{M}$. Why women choose compounded bioidentical hormone therapy: lessons from a qualitative study of menopausal decision-making. MC Womens Health 2017; 17(1):97

9. NICE guidelines, accessed 4/24/2020 https://www.nice.org.uk/guidance/ng23/chapter/Recommendations\#managing-short-term-menopausal-symptoms

10. Sarrell PM, Niike VY, Vinante V, et al. The Mortality Toll of Estrogen Avoidence, An analysis of excess death in hysterectomized women aged 50 to 59 years. Am J Public Health. 2013; 103(9):1583-8.

11. Manson JE, Aragaki AK, Rossouw JE. Menopausal Hormone Therapy and Long-term All-Cause and Cause-Specific Mortality: The Women's Health Initiative Randomized Trials. JAMA. 2017;318 (10):927-938.

12. Manson JE, Kaunitz AM. Menopause Management - Getting Clinical Care Back on Track. NEJM. 2016;374(9):803-6.

13. Christianson MS, Ducie JA, Altman $\mathbf{K}$, et al. Menopause Education: Needs Assessment of American Obstetrics and Gynecology Residents. Menopause. 2013;20(11): 1120-5

14. Hersh AL, Stefanick ML, Stafford RS. National Use of Postmenopausal Hormone Therapy: Annual Trends and Response to Recent Evidence. JAMA. 2004:291(1):47-53.

15. Kling et al. Menopause Management Knowledge in Postgraduate Family Medicine, Internal Medicine, and Obstetrics and Gynecology Residents: A Cross-Sectional Survey. Mayo Clin Proc. 2019. Volume 94, Issue 2, Pages 242-253.

16. Jack G, Riach K, Bariola E. et al. Menopause in the workplace: What employers should be doing. Maturitas 2016; 85:88-95.

17. Salpeter S.R., Walsh J.M., Ormiston T.M. et al. Meta-analysis: effect of hormone-replacement therapy on components of the metabolic syndrome in postmenopausal women. Diabetes Obes Metab 2006;8(5)538-554.

Publisher's Note: Springer Nature remains neutral with regard to jurisdictional claims in published maps and institutional affiliations. 